\title{
Hydraulic Model of the Skin Friction Reduction with Surface Grooves
}

\author{
Bettina Frohnapfel ${ }^{1}$, Peter Lammers ${ }^{2}$, Jovan Jovanovic ${ }^{1}$ and Antonio Delgado ${ }^{1}$ \\ ${ }^{1}$ Institute of Fluid Mechanics, Friedrich-Alexander University \\ Erlangen-Nuremberg, Cauerstr. 4, 91058 Erlangen, Germany; \\ E-mail: bettina@lstm.uni-erlangen.de \\ ${ }^{2}$ High Performance Computing Center Stuttgart, Nobelstr. 19, 70569 Stuttgart, \\ Germany
}

\begin{abstract}
The reduction of skin friction in turbulent flows holds considerable promise for energy savings. The present work shows how and why skin friction and the dissipation are interrelated in turbulent channel flows. A hydraulic model formulation is presented for the skin friction reduction that can be obtained with a surface structure recently proposed for flow control. The model predictions are validated with results from direct numerical simulations.
\end{abstract}

Key words: Flow control, skin friction reduction, surface structures.

\section{Skin Friction Reduction}

It is well known that the skin friction coefficient

$$
c_{f}=\frac{\tau_{w}}{\frac{\rho}{2} U_{b}^{2}}
$$

(where $\tau_{w}$ is the wall shear stress and $U_{b}$ the bulk flow velocity) suddenly increases when laminar to turbulent transition occurs. The transition delay or the reduction of the skin friction in turbulent flows therefore holds significant potential for energy savings. Employing the momentum and energy conservation for a turbulent channel flow of channel height $H$, it can be shown that $c_{f}$ is related to the average total dissipation $\bar{\varepsilon}$ by:

$$
\bar{\varepsilon} \simeq c_{f} \frac{U_{b}^{3}}{H},
$$

where $\bar{\varepsilon}$ is given as follows: 


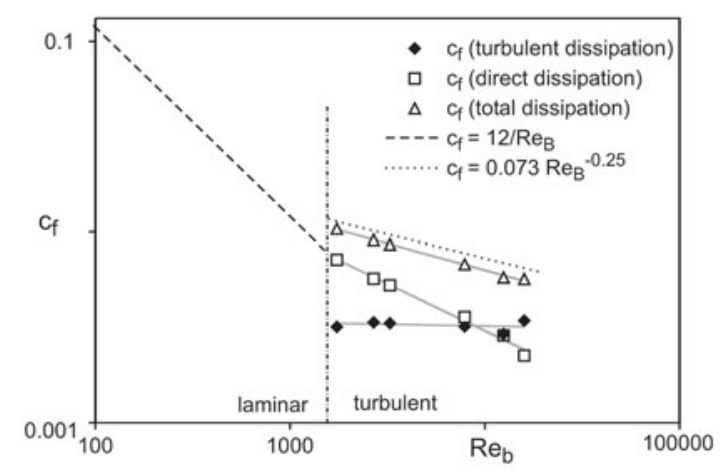

Fig. 1 Friction coefficient as a function of bulk Reynolds number in the laminar and turbulent flow regimes.

$$
\bar{\varepsilon}=\frac{1}{V} \int_{V} \underbrace{v\left(\frac{\partial \bar{U}_{1}}{\partial x_{2}}\right)^{2}}_{\text {direct dissipation }}+\underbrace{v \frac{\overline{\partial u_{j}}}{\partial x_{i}} \frac{\partial u_{j}}{\partial x_{i}}}_{\text {turbulent dissipation }} d V
$$

Figure 1 shows a plot of $c_{f}$, based on $\bar{\varepsilon}$, versus Reynolds number, $R e_{b}=U_{b} H / v$ for turbulent channel flows at different Reynolds numbers [1,5,6,8,9] including the theoretical result for a laminar channel flow:

$$
c_{f}=\frac{12}{R e_{b}}
$$

and the typical correlation for a turbulent channel flow [2]:

$$
c_{f}=0.073 R e_{b}^{-0.25}
$$

It can clearly be seen that an increase of $c_{f}$ at high Reynolds number is caused by turbulent dissipation. In order to obtain energy savings in the high Reynolds number regime, it is therefore necessary to reduce the turbulent dissipation. In [3] it is shown that the turbulent dissipation can be significantly reduced by forcing the turbulent fluctuations in the near-wall region to be predominantly one-component. In order to realize this state of turbulence at the wall a surface topology with grooves aligned in the mean flow direction as shown in Figure 2(left) is proposed. Inside the grooves, fluctuations in the spanwise direction are suppressed such that their intensity is almost identical to the one found in the wall-normal direction. The fluctuations in the streamwise direction are not restricted to grow by the surface topology and are therefore the most dominant ones inside the grooves. 


\subsection{Formulation of the Hydraulic Model}

To estimate the performance of the proposed surface a hydraulic model is formulated. In [4] it is shown that a trend towards one-component turbulence is commonly found for different existing drag reduction techniques and that this trend resembles the one for decreasing Reynolds numbers in uncontrolled turbulent channel flows. Based on these results it can be concluded that we may expect relaminarization of a turbulent flow near the wall if two of the three velocity components are fully suppressed. The hydraulic model formulation is therefore based on the assumption that the flow inside the grooves will laminarize if these are of the same order as the sublayer thickness. The skin friction within the grooves is given by Equation (4), with the Reynolds number based on the characteristic length and velocity scales (which are on the order of the groove dimensions) and the wall shear velocity on the wall sections between the grooves where Equation (5) holds. Thus the main contribution to the skin friction arises from the wall sections which separate the grooves from each other. The resulting drag reduction $D R$ for the grooved surface is therefore given by:

$$
D R=1-\frac{\tau_{w, \text { grooved }} A_{\text {grooved }}}{\tau_{w, \text { smooth }} A_{\text {smooth }}},
$$

where $\tau_{w}$ is the wall shear stress of the grooved surface structure and the smooth surface of a regular turbulent channel flow, respectively, and $A$ denotes the corresponding surface areas. Figure 2 (right) shows the model predictions for a surface structure in which the grooves are separated by a distance of three times their width $(b=3 a)$. Drag reduction asymptotically tends to $25 \%$ for very high Reynolds numbers when the skin friction inside the grooves can be neglected:

$$
D R_{\max } \approx 1-\frac{b}{a+b}=\frac{a}{a+b}
$$

\subsection{Comparison with Computational Results}

In order validate the model description, the obtained drag reduction is compared with results of direct numerical simulations of a channel flow with grooved surfaces. These simulations are based on the lattice-Boltzmann method. A detailed description of the employed simulation technique can be found in [7] where the applicability and accuracy of this method for channel flow simulations is discussed in detail. Simulations were carried out at two different Reynolds numbers; the details of the simulations are given in Table 1. Based on the obtained velocity profiles, the wall shear stress is calculated and the resulting drag reduction is obtained by comparing with a channel flow without grooves (Equation (6)). The results are included in Figure 2 (right) and show good agreement with the model predictions. 
Table 1 Overview of the simulations carried out for the channel flow with grooves where $\operatorname{Re}_{c}$ is the Reynolds number based on centerline velocity and $R e_{\tau}$ the one based on the wall shear velocity.

\begin{tabular}{ccccccc}
\hline \multirow{2}{*}{$R e_{c}$} & \multirow{2}{*}{$e_{\tau}$} & resolution & resolution & $a^{+}$ & $b^{+}$ & $c^{+}$ \\
\cline { 5 - 7 } & & $N 1 \times N 2 \times N 3$ & in wall units & & in wall units & \\
\hline 6650 & 187 & $4096 \times 264 \times 240$ & 1.4 & 5 & 14 & 5 \\
5230 & 148 & $4096 \times 364 \times 360$ & 0.8 & 4 & 15 & 4 \\
\hline
\end{tabular}

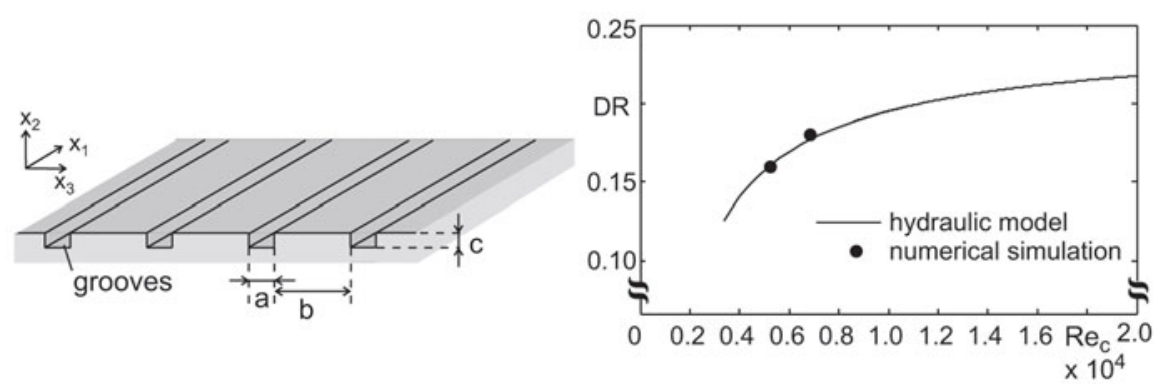

Fig. 2 Surface structure with grooves aligned in the flow direction (left). Drag reduction of the grooved surface based on the hydraulic model in comparison with numerical results (right).

\section{Outlook and Conclusion}

A hydraulic model is presented for the drag reducing performance of a grooved surface structure which was designed to minimize the turbulent dissipation in the flow. The obtained model predictions show very good agreement with the drag reduction obtained in direct numerical simulations. Future work will concentrate on experimental verification [10] of the obtained drag reduction and more refined numerical investigations.

\section{References}

1. R.A. Antonia, M. Teitel, J. Kim, L.W.B. Browne: Low-Reynolds-number effects in a fully developed turbulent channel flow. J. Fluid Mech. 236 (1992) 579-605.

2. R.B. Dean: Reynolds number dependence of skin friction and other bulk flow variables in two-dimensional rectangular duct flow. J. Fluids Engrg. 100 (1978) 215-223.

3. B. Frohnapfel, P. Lammers, J. Jovanović: The role of turbulent dissipation for flow control of near-wall turbulence. In Notes on Numerical Fluid Mechanics and Multidisciplinary Design, C. Tropea, S. Jarkilic, H.-J. Heinemann, R. Henke, H. Hönlinger (Eds.), Springer, Berlin (2007) in print.

4. B. Frohnapfel, P. Lammers, J. Jovanović, F. Durst: Interpretation of the mechanism associated with turbulent drag reduction in terms of anisotropy invariants. J. Fluid Mech. 577 (2007) $457-466$. 
5. J. Kim, P. Moin, R. Moser: Turbulence statistics in a fully developed channel flow at low Reynolds numbers. J. Fluid Mech. 177 (1987) 133-166.

6. A. Kuroda, N. Kasagi, M. Hirata: A direct numerical simulation of the fully developed turbulent channel flow. In Proceedings of International Symposium on Computational Fluid Dynamics, Nagoya, Japan (1989) pp. 1174-1179.

7. P. Lammers: Direct numerical simulations of wall-bounded flows at low Reynolds number with the lattice-Boltzmann method. Ph.D. Thesis, University of Erlangen-Nuremberg (2004) [in German].

8. R.D. Moser, J. Kim, N.N. Mansour: Direct numerical simulation of turbulent channel flow up to $R e_{\tau}=590$. Phys. Fluids 11 (1999) 943-945.

9. R. Volkert: Determination of statistical turbulence quantities for a turbulent channel flow based on direct numerical simulations. Ph.D. Thesis, University of Erlangen-Nuremberg (2006) [in German].

10. B. Frohnapfel, J. Jovanović, A. Delgado: Experimental investigation of turbulent drag reduction by surface embedded grooves. J. Fluid Mech. (2007) in print. 